TRANSACTIONS OF THE

AMERICAN MATHEMATICAL SOCIETY

Volume 359, Number 12, December 2007, Pages 6111-6126

S 0002-9947(07)04261-4

Article electronically published on June 4, 2007

\title{
GÉNÉRALISATION DU CRITÈRE DE BEURLING-NYMAN POUR L'HYPOTHĖSE DE RIEMANN
}

\author{
ANNE DE ROTON
}

\begin{abstract}
Nous généralisons dans cet article le critère de Beurling-Nyman, qui concerne la fonction $\zeta$ de Riemann, à une large classe de séries de Dirichlet. Nous établissons donc une correspondance entre la densité d'un certain sousespace de fonctions dans $L^{2}(0,1)$ et la localisation des zéros d'une série de Dirichlet. Nous utilisons pour obtenir ce résultat la structure de l'espace de Hardy du demi-plan.

Abstract. We generalise Beurling-Nyman's criterion, already known for the Riemann $\zeta$ function, to a larger class of Dirichlet series. We reveal a link between the density of some subspace of functions in $L^{2}(0,1)$ and the localization of the zeros of a Dirichlet series. To do so, we use the structure of the Hardy space of the half-plan.
\end{abstract}

\section{INTRODUCTION}

Les travaux de Szàsz $\mathrm{Sz}$ d'abord, Paley et Wiener PW, Levinson Lev et Schwartz $[\mathrm{Sch}]$ ensuite, mettent en lumière des relations entre la densité de certains sous-espaces fonctionnels et la répartition des zéros des fonctions analytiques. Cette idée a été exploitée pour les zéros de la fonction $\zeta$ par Nyman dans sa thèse puis par Beurling dans $\mathrm{Be}$. Dans ce dernier article, A. Beurling introduit l'ensemble $\widetilde{\mathcal{B}}$ des fonctions complexes $f$ définies sur l'intervalle ]0,1] par :

$$
f(t):=\sum_{k=1}^{n} a_{k}\left\{\frac{\theta_{k}}{t}\right\},
$$

où $n \geq 1,0<\theta_{k} \leq 1, a_{k} \in \mathbb{C}, \sum_{k=1}^{n} a_{k} \theta_{k}=0$ et $\{x\}$ désigne la partie fractionnaire de $x$. Le résultat de Nyman, exposé par Beurling dans [Be], s'énonce ainsi :

Théorème 1.1 (Nyman). Les trois assertions suivantes sont équivalentes:

(1) $\zeta(s)$ ne s'annule pas dans le demi-plan $\Re s>1 / 2$;

(2) $\widetilde{\mathcal{B}}$ est dense dans $L^{2}(0,1)$;

(3) la fonction constante égale à -1 est dans l'adhérence de $\widetilde{\mathcal{B}}$ dans $L^{2}(0,1)$.

Ce résultat est analysé et commenté dans [BS]. Il a été complété par Beurling dans $\mathrm{Be}$, Bercovici et Foias dans $\mathrm{BF}$. Ces trois auteurs établissent une correspondance similaire entre la densité de $\widetilde{\mathcal{B}}$ dans $L^{p}(0,1)$ et l'absence de zéros de la

Received by the editors September 2, 2004 and, in revised form, January 6, 2006.

2000 Mathematics Subject Classification. Primary 37A45.

Key words and phrases. Séries de Dirichlet, critère de Beurling-Nyman, hypothèse de Riemann généralisée, classe de Selberg, espace de Hardy.

(C)2007 American Mathematical Society Reverts to public domain 28 years from publication 
fonction $\zeta$ dans le demi-plan $\Re s>1 / p$. Le cas $p=1$ est traité dans $\overline{\mathrm{BF}}$, les autres cas dans $[\mathrm{Be}$.

Dans cet article, nous généralisons le résultat de Nyman à une classe de fonctions $F$ comprenant la fonction $\zeta$ de Riemann.

Nous définirons un sous-espace $\widetilde{\mathcal{B}_{F}}$ de $L^{2}(0,1)$ et nous montrerons que sa densité dans $L^{2}(0,1)$ caractérise la non-annulation de la fonction $F$ dans le demi-plan $\{\Re s>1 / 2\}$. Pour cela, nous étudierons l'image de $\widetilde{\mathcal{B}_{F}}$ par la transformation de Mellin. Nous montrerons que c'est un sous-espace de l'espace de Hardy du demi-plan $\{\Re s>1 / 2\}$ invariant par l'action du semi-groupe des contractions. L'utilisation des propriétés des espaces de Hardy et plus particulièrement du théorème de factorisation et du théorème de Beurling-Lax nous permettra de conclure.

La principale difficulté de ce travail de généralisation consiste à choisir un sousespace $\widetilde{\mathcal{B}_{F}}$ qui convienne et plus précisément à choisir pour la fonction $F$ une fonction $\Psi_{F}$ qui joue le même rôle que la partie fractionnaire pour la fonction $\zeta$.

De plus, la démonstration de notre théorème nécessite une hypothèse : la fonction $\Psi_{F}$ doit appartenir à l'espace $L^{2}(] 0,+\infty\left[, \frac{d t}{t^{2}}\right)$. On peut supprimer cette hypothèse dans certains cas, mais c'est au prix d'hypothèses supplémentaires sur la fonction $F$. Nous avons donc dans un premier temps démontré un théorème s'appliquant à une large classe de séries de Dirichlet sous l'hypothèse $\Psi_{F} \in L^{2}(] 0,+\infty\left[, \frac{d t}{t^{2}}\right)$; nous avons ensuite spécifié notre théorème pour des fonctions de la classe de Selberg, le reliant ainsi à l'hypothèse de Riemann généralisée. Dans ce dernier cas, nous montrons que l'on peut supprimer l'hypothèse faite sur $\Psi_{F}$ en utilisant une version lissée de cette fonction.

\section{RAPPELS}

Définition 2.1. Nous désignerons par $H^{2}$ l'espace de Hardy du demi-plan $\{\Re s>$ $1 / 2\}$, i.e. l'ensemble des fonctions $f(s)$, holomorphes dans le demi-plan $\{\Re s>1 / 2\}$, vérifiant:

$$
\|f\|^{2}=\sup _{x>1 / 2} \int_{\Re s=x}|f(s)|^{2}|d s|<+\infty .
$$

Définition 2.2. On appellera produit de Blaschke toute fonction de la forme

$$
B_{Z}(s)=\prod_{\beta \in Z} \frac{|\beta(1-\beta)|}{\beta(1-\beta)} \frac{s-\beta}{s+\bar{\beta}-1},
$$

où $Z$ est un multi-ensemble de points du demi-plan $\{\Re s>1 / 2\}$ tel que

$$
\sum_{\beta \in Z} \frac{\Re(\beta-1 / 2)}{|\beta|^{2}}<+\infty
$$

et avec la convention $\frac{|\beta(1-\beta)|}{\beta(1-\beta)}=1$ pour $\beta=1$.

On appellera fonction intérieure singulière toute fonction de la forme

$$
S_{\mu, v}(s)=\exp \left(-v\left(s-\frac{1}{2}\right)-\int_{-\infty}^{+\infty} \frac{2 i t s-\left(\frac{1}{2}+i t\right)}{\frac{1}{2}+i t-s} d \mu(t)\right)
$$

avec $v \in \mathbb{R}^{+}, \mu$ mesure positive finie sur la droite réelle, singulière à la mesure de Lebesgue. 
Si $\omega$ est une fonction positive de $L^{2}(\mathbb{R})$ telle que $\int_{-\infty}^{+\infty} \frac{\log \omega(t)}{1+t^{2}} d t>-\infty$, on définit

$$
O_{\omega}(s)=\exp \left(\frac{1}{2 \pi} \int_{-\infty}^{+\infty} \frac{2 i t s-\left(\frac{1}{2}+i t\right)}{\frac{1}{2}+i t-s} \frac{\log \omega(t)}{\frac{1}{4}+t^{2}} d t\right) .
$$

Nous sommes à présent en mesure d'énoncer le théorème de factorisation dans $H^{2}$. C'est la formule de factorisation de l'article [BS].

Théorème 2.3. Soit $f$ une fonction de $H^{2}$ non nulle. Alors il existe un unique quintuplet $(c, Z, \mu, v, \omega)$ vérifiant :

$-c \in \mathbb{C},|c|=1$,

- $Z$ est un multi-ensemble du demi-plan $\{\Re s>1 / 2\}$ satisfaisant (2.1),

- $\mu$ est une mesure positive finie sur la droite réelle, singulière à la mesure de Lebesgue,

- $v$ est un réel positif ou nul,

- $\omega$ est une fonction positive de $L^{2}(\mathbb{R})$ telle que $\int_{-\infty}^{+\infty} \frac{\log \omega(t)}{1+t^{2}} d t>-\infty$,

tel que

$$
f=c B_{Z} S_{\mu, v} O_{\omega}
$$

De plus, $Z$ est le multi-ensemble des zéros de $f$ dans le demi-plan $\{\Re s>1 / 2\}$ comptés avec leur multiplicité, le support de $\mu$ est inclus dans l'ensemble des $t$ tels que $\frac{1}{2}+$ it est un point singulier de $f$, et pour $x \geq 1, f(x) \ll e^{-v x}$.

Le groupe des dilatations/contractions $\left\{D_{\theta}, \theta>0\right\}$ agit sur les fonctions $g$ de $L^{2}(0,+\infty)$ par :

$$
D_{\theta} g(t)=\frac{1}{\sqrt{\theta}} g\left(\frac{t}{\theta}\right)
$$

Le semi-groupe des contractions $\left\{D_{\theta}, 0<\theta \leq 1\right\}$ agit de même sur les fonctions de $L^{2}(0,1)$ et sur les fonctions de $H^{2}$ par :

$$
D_{\theta} G(s)=\theta^{s-\frac{1}{2}} G(s) .
$$

Définition 2.4. Un opérateur $T$ sur un sous-espace de $L^{2}(0,+\infty)$ sera dit invariant s'il commute avec les dilatations/contractions, i.e. si pour tout $\theta>0, D_{\theta} T=T D_{\theta}$.

On dira qu'un sous-espace de $L^{2}(0,1)$ ou de $H^{2}$ est invariant s'il est invariant par l'action du semi-groupe des contractions.

Introduisons à présent la transformation de Mellin.

Définition 2.5. Soit $] a, b[$ un intervalle non vide de $\mathbb{R}$ et soit $f:] 0,+\infty[\rightarrow \mathbb{C}$, Lebesgue-mesurable et telle que, pour tout $\sigma \in] a, b[$,

$$
\int_{0}^{+\infty}|f(x)| x^{\sigma-1} d x<+\infty
$$

On appellera transformée de Mellin de $f$ et on notera $\mathcal{M} f$ la fonction définie dans la bande verticale $\{a<\Re s<b\}$ par

$$
\mathcal{M} f(s)=\int_{0}^{+\infty} f(t) t^{s-1} d t
$$

Notations 1 . On écrira $L^{2}(0,1)$ pour l'ensemble des fonctions de $L^{2}(0,+\infty)$ identiquement nulles sur $] 1,+\infty[$. 
Si $f$ est une fonction de $L^{2}(0,1)$, alors pour tout $\sigma>1 / 2$, l'intégrale

$$
\int_{0}^{+\infty}|f(x)| x^{\sigma-1} d x
$$

converge et on peut donc définir la transformée de Mellin de $f$ dans le demi-plan $\{\Re s>1 / 2\}$. Le théorème de Paley-Wiener affirme que la transformation de Mellin $\mathcal{M}$ est une isométrie (à une constante près avec nos conventions) de $L^{2}(0,1)$ dans $H^{2}$. D'autre part, il est facile de vérifier qu'un sous-espace de $L^{2}(0,1)$ est invariant si et seulement si l'image de ce sous-espace par la transformation de Mellin est un sous-espace invariant de $H^{2}$.

Théorème 2.6 (Théorème de Beurling et Lax). Soit $\left(F_{\alpha}\right)_{\alpha \in A}$ une famille de fonctions de $H^{2}$. Alors le plus petit sous-espace fermé invariant de $H^{2}$ et contenant tous les $F_{\alpha}, \alpha \in A$ est $B_{Z} S_{\mu, v} H^{2}$ avec $Z=\bigcap_{\alpha \in A} Z\left(F_{\alpha}\right), \mu=\inf _{\alpha \in A} \mu\left(F_{\alpha}\right), v=$ $\inf _{\alpha \in A} v\left(F_{\alpha}\right)$.

Ce théorème est le théorème 2 de l'article $[\mathrm{BS}]$.

\section{GÉnéralisation du théorème de Beurling-Nyman}

Hypothèses 1. Dans cette partie, nous supposerons que $F(s)=\sum_{n=1}^{\infty} \frac{a(n)}{n^{s}}$ est une série de Dirichlet absolument convergente pour $\Re s>1$ admettant un prolongement méromorphe au demi-plan $\Re s \geq \frac{1}{2}$ avec un unique pôle éventuel d'ordre fini en $s=1$. On supposera de plus que pour tout $\varepsilon>0, a_{n}=O\left(n^{\varepsilon}\right)$.

Notations 2. On notera $m_{F}$ l'ordre polaire de $F$ en $s=1$.

3.1. Fonction complémentaire. Dans le cas de la fonction $\zeta$ de Riemann, la démonstration du théorème de Beurling-Nyman repose sur la formule

$$
\int_{0}^{+\infty}\left\{\frac{1}{t}\right\} t^{s-1} d t=-\frac{\zeta(s)}{s}, 0<\Re s<1,
$$

qui relie la fonction partie fractionnaire à la fonction $\zeta$ via la transformation de Mellin. Afin d'obtenir une formule analogue à (3.1), nous introduisons la fonction complémentaire associée à la fonction $F$.

Définition 3.1. Soit $F$ une fonction vérifiant les hypothèses 1. On définit la fonction complémentaire associée à $F$ par :

$$
\begin{aligned}
\Psi_{F}: \mathbb{R}^{+} & \rightarrow \mathbb{C} \\
x & \mapsto \operatorname{Res}\left(\frac{x^{s}}{s} F(s), 1\right)-\sum_{n \leq x} a_{n} .
\end{aligned}
$$

On définit également la fonction $\Psi_{F}^{(1)} \operatorname{par} \Psi_{F}^{(1)}(x)=\Psi_{F}(1 / x)$.

Notations 3. Notons $A(s):=\sum_{k=1}^{m_{F}} \frac{p_{-k}}{(s-1)^{k}}$ la partie polaire de $F(s) / s$ en $s=1$.

On peut montrer que

$$
\operatorname{Res}\left(\frac{x^{s}}{s} F(s), 1\right)=x P_{F}(\ln x) \text { où } P_{F} \text { est le polynôme } P_{F}(X)=\sum_{l=0}^{m_{F}-1} p_{-l-1} \frac{X^{l}}{l !} \text {. }
$$

La proposition suivante permet de relier la fonction complémentaire associée à $F$ à la fonction $F$ via la transformation de Mellin, justifiant à posteriori l'introduction de la fonction $\Psi_{F}$. 
Proposition 3.1. Soit $F$ une fonction vérifiant les hypothèses 1. Si $\Psi_{F}^{(1)}$ est une fonction de $L^{2}(0,+\infty)$, alors pour $\frac{1}{2}<\Re s<1$, on a :

$$
\mathcal{M} \Psi_{F}^{(1)}(s)=-\frac{F(s)}{s} .
$$

Démonstration. Pour $\Re s>1$, en notant $\Sigma_{F}(t)=\sum_{n \leq t} a_{n}$, on a

$$
F(s)=\sum_{n \geq 1} a_{n} n^{-s}=\int_{1^{-}}^{+\infty} t^{-s} d \Sigma_{F}(t)=s \int_{1}^{+\infty} t^{-s-1} \Sigma_{F}(t) d t
$$

car d'après les hypothèses sur $F, \Sigma_{F}(t)=O\left(t^{1+\varepsilon}\right)$ pour tout $\varepsilon>0$. Ainsi

$$
F(s)=s \int_{1}^{+\infty} t^{-s} P_{F}(\ln t) d t-s \int_{1}^{+\infty} \Psi_{F}(t) t^{-s-1} d t .
$$

Or, pour $\Re s>1$,

$$
\int_{1}^{+\infty} P_{F}(\ln t) t^{-s} d t=A(s)
$$

et la fonction $\frac{F(s)}{s}-A(s)$ est holomorphe pour $\Re s>1 / 2$. Comme $\Psi_{F}^{(1)} \in L^{2}(0,+\infty)$, l'intégrale $\int_{0}^{1} \Psi_{F}^{(1)}(u) u^{s-1} d u$ converge et définit une fonction de la variable $s$ holomorphe pour $\Re s>1 / 2$ et donc si $\Re s>1 / 2$, par unicité du prolongement analytique on a :

$$
-\frac{F(s)}{s}+A(s)=\int_{1}^{+\infty} \Psi_{F}(t) t^{-s-1} d t=\int_{0}^{1} \Psi_{F}^{(1)}(u) u^{s-1} d u .
$$

Par ailleurs, pour $\Re s<1$,

$$
\int_{0}^{1} \Psi_{F}(t) t^{-s-1} d t=\int_{0}^{1} P_{F}(\ln t) t^{-s} d t=-A(s)
$$

Pour $1 / 2<\Re s<1$, en utilisant (3.4) et (3.5), on obtient :

$$
-\frac{F(s)}{s}=\int_{0}^{+\infty} \Psi_{F}(t) t^{-s-1} d t=\int_{0}^{+\infty} \Psi_{F}^{(1)}(t) t^{s-1} d t .
$$

Remarque 1. La condition $\Psi_{F}^{(1)} \in L^{2}(0,+\infty)$ assure la convergence uniforme de l'intégrale de droite dans tout compact du demi-plan $\Re s>1 / 2$ et garantit donc la validité de l'égalité (3.4) dans ce même demi-plan.

3.2. Les sous-espaces $\mathcal{B}_{F}$ et $\widetilde{\mathcal{B}_{F}}$. Nous introduisons à présent les espaces dont la densité dans $L^{2}(0,1)$ va, sous certaines hypothèses, caractériser la non-annulation de la fonction $F$ dans le demi-plan $\{\Re s>1 / 2\}$.

Définition 3.2. On définit les espaces de fonctions suivants :

$$
\mathcal{B}_{F}=\left\{f: t \mapsto \sum_{k=1}^{n} c_{k} \Psi_{F}\left(\frac{\alpha_{k}}{t}\right), n \in \mathbb{N}, \forall k \in[1, n], c_{k} \in \mathbb{C}, 0<\alpha_{k} \leq 1\right\}
$$

et

où

$$
\widetilde{\mathcal{B}_{F}}=\left\{f: t \mapsto \sum_{k=1}^{n} c_{k} \Psi_{F}\left(\frac{\alpha_{k}}{t}\right) \in \mathcal{B}_{F}, c_{k} \text { et } \alpha_{k} \text { vérifiant (플 }\right\}
$$

$$
\forall l \in\left[\left[0, m_{F}-1\right]\right], \sum_{k=1}^{n} c_{k} \alpha_{k}\left(\ln \alpha_{k}\right)^{l}=0
$$


Remarque 2. La condition (A (A) est une condition nécessaire et suffisante pour que la fonction $f(t)=\sum_{k=1}^{n} c_{k} \Psi_{F}\left(\frac{\alpha_{k}}{t}\right)$ soit identiquement nulle sur $[1,+\infty[$. Si cette condition est vérifiée, alors 1 est un zéro de la fonction $g$ définie sur $\mathbb{C}$ par $g(s):=$ $\sum_{k=1}^{n} c_{k} \alpha_{k}^{s}$, avec une multiplicité supérieure ou égale à $m_{F}$.

Proposition 3.2. Soit $F$ une fonction vérifiant les hypothèses 1. Si on suppose de plus que $\Psi_{F}^{(1)} \in L^{2}(0,+\infty)$, alors on a:

$$
\overline{\widetilde{\mathcal{B}_{F}}}=\left\{f \in L^{2}(0,1), \frac{\mathcal{M} f(s)}{F(s)} \text { holomorphe pour } \Re s>\frac{1}{2}\right\} .
$$

Démonstration. Notons $K$ l'espace

$$
K=\left\{f \in L^{2}(0,1), \frac{\mathcal{M} f(s)}{F(s)} \text { holomorphe pour } \Re s>\frac{1}{2}\right\} .
$$

Remarquons dans un premier temps que grâce au théorème de Paley-Wiener, on a $K=\mathcal{M}^{-1}\left(B_{Z_{F}} H^{2}\right)$ où $Z_{F}$ désigne le multi-ensemble des zéros de $F$ comptés avec leur multiplicité dans le demi-plan $\{\Re s>1 / 2\}$ et $B_{Z_{F}}$ le produit de Blaschke défini à partir de $Z_{F}$.

Notons que $\widetilde{\mathcal{B}_{F}}$ est un sous-espace invariant de $L^{2}(0,1)$.

En effet, soient $g(t)=\sum_{k=1}^{n} c_{k} \sqrt{\alpha_{k}} D_{\alpha_{k}} \Psi_{F}^{(1)}(t) \in \widetilde{\mathcal{B}_{F}}$ et $\left.\left.\theta \in\right] 0,1\right]$. Alors :

$$
D_{\theta} g(t)=\sum_{k=1}^{n} c_{k} \sqrt{\alpha_{k}} D_{\theta \alpha_{k}} \Psi_{F}^{(1)}(t)=\sum_{k=1}^{n} c_{k} \frac{1}{\sqrt{\theta}} \Psi_{F}\left(\frac{\theta \alpha_{k}}{t}\right) .
$$

De plus, comme pour tout entier $l<m_{F}$, on a $\sum_{k=1}^{n} c_{k} \alpha_{k} \ln ^{l}\left(\alpha_{k}\right)=0$, alors on a $\sum_{k=1}^{n} c_{k} \frac{1}{\sqrt{\theta}} \alpha_{k} \theta \ln ^{l}\left(\theta \alpha_{k}\right)=0$.

On en déduit grâce au théorème de Paley-Wiener que $\mathcal{\mathcal { M }} \widetilde{\widetilde{\mathcal{B}_{F}}}$ est un sous-espace fermé invariant de $H^{2}$.

Nous allons maintenant préciser la forme des fonctions de l'espace $\mathcal{M} \widetilde{\mathcal{B}_{F}}$.

Soit $g(t)=\sum_{k=1}^{n} c_{k} \Psi_{F}\left(\frac{\alpha_{k}}{t}\right)$ une fonction de $\widetilde{\mathcal{B}_{F}}$. D'après le théorème 2.3, la fonction $G=\mathcal{M} g \in H^{2}$, comme tout élément non nul de $H^{2}$, se factorise comme suit :

$$
G(s)=c_{G} B_{Z_{G}}(s) S_{\mu_{G}, v_{G}}(s) O_{w_{G}}(s),
$$

où $\left(c_{G}, Z_{G}, \mu_{G}, v_{G}, w_{G}\right)$ est le quintuplet associé à $G$.

D'autre part,

$$
G(s)=\mathcal{M} g(s)=-\frac{F(s)}{s} \sum_{k=1}^{n} c_{k} \alpha_{k}^{s},
$$

et la fonction $F$ n'a pas de singularité sur la droite $\Re s=\frac{1}{2}$ donc $G$ non plus et donc (voir théorème 2.3) la mesure $\mu_{G}$ est nulle.

Examinons maintenant le comportement de $G$ sur la demi-droite réelle ]1, $+\infty[$ au voisinage de l'infini. Supposons les $\alpha_{k}$ distincts deux à deux et associés à des coefficients $c_{k}$ non nuls et posons $\alpha_{i}=\max _{k=1}^{n} \alpha_{k}$. On a

$$
|G(x)|=\frac{|F(x)|}{x}\left|\sum_{k=1}^{n} c_{k} \alpha_{k}^{x}\right| \asymp \frac{1}{x}\left|c_{i}\right| e^{x \ln \alpha_{i}} \asymp \frac{\left|c_{i}\right|}{x} e^{-x\left|\ln \alpha_{i}\right|} .
$$

D'autre part, d'après le théorème 2.3 , pour $x \geq 1$, on a $G(x) \ll e^{-v_{G} x}$.

Ainsi, $v_{G} \leq\left|\ln \alpha_{i}\right|$. Or on peut choisir $g \in \widetilde{\mathcal{B}_{F}}$ telle que $\alpha_{1}=1$, donc $v_{G}=0$. 
Pour $G \in \mathcal{M}\left(\overline{\widetilde{\mathcal{B}_{F}}}\right)$, notons $Z_{G}$ l'ensemble des zéros de la fonction $G$ dans le demi-plan $\Re s>\frac{1}{2}$ et notons

$$
Z=\bigcap_{G \in \mathcal{M}\left(\overline{\overline{\mathcal{B}_{F}}}\right)} Z_{G} .
$$

D'après (3.6), il est clair que $Z_{F} \subset Z$.

De plus, pour tout $s_{1} \neq 1$, il existe une fonction $g_{1} \in \widetilde{\mathcal{B}_{F}}$ telle que $\sum_{k=1}^{n} c_{k} \alpha_{k}^{s_{1}} \neq 0$ et il existe $g_{2} \in \widetilde{\mathcal{B}_{F}}$ telle que la multiplicité du zéro 1 de la fonction $\sum_{k=1}^{n} c_{k} \alpha_{k}^{s}$ soit exactement égale à $m_{F}$. Ainsi on a bien $Z=Z_{F}$.

Le sous-espace $\mathcal{M}\left(\overline{\widetilde{\mathcal{B}_{F}}}\right)$ est le plus petit sous espace fermé de $H^{2}$, invariant, contenant tous les $-\frac{F(s)}{s} \sum_{k=1}^{n} c_{k} \alpha_{k}^{s}, c_{k}$ et $\alpha_{k}$ vérifiant les conditions (A). L'espace $\mathcal{M}\left(\widetilde{\widetilde{\mathcal{B}_{F}}}\right)$ est donc, d'après le théorème 2.6 , le sous-espace $B_{Z} S_{\mu, v} H^{2}$ avec $Z=$ $\bigcap_{g \in \overline{\overline{\mathcal{B}_{F}}}} Z_{\mathcal{M} g}=Z_{F}, \mu=\inf _{g \in \overline{B_{F}}} \mu_{\mathcal{M} g}=0, v=\inf _{g \in \overline{B_{F}}} v_{\mathcal{M} g}=0$.

On a donc $B_{Z_{F}} H^{2}=\mathcal{M}\left(\overline{\widetilde{\mathcal{B}_{F}}}\right)$, ce qui permet de conclure.

Remarque 3. Ici la condition $\Psi_{F}^{(1)} \in L^{2}(0,+\infty)$ est cruciale. Elle permet de travailler avec un sous-espace de $L^{2}(0,1)$ et donc d'utiliser le théorème de PaleyWiener, passage essentiel de cette démonstration.

3.3. Le théorème de Beurling-Nyman généralisé. Nous sommes maintenant en mesure d'énoncer le critère de Beurling-Nyman généralisé.

Théorème I. Soit $F$ une fonction vérifiant les hypothèses 1 telle que $\Psi_{F}^{(1)} \in$ $L^{2}(0,+\infty)$. Alors les assertions suivantes sont équivalentes :

(1) La fonction $F$ ne s'annule pas dans le demi-plan $\Re s>1 / 2$;

(2) le sous-espace $\widetilde{\mathcal{B}_{F}}$ est dense dans $L^{2}(0,1)$;

(3) la fonction indicatrice $\chi$ de l'intervalle ]0, 1] appartient à l'adhérence de $\widetilde{\mathcal{B}_{F}}$ dans $L^{2}(0,1)$.

Démonstration. D'après la proposition [3.2, les deux premières assertions sont équivalentes et il est d'autre part clair que la seconde assertion entraîne la troisième. Il nous suffit donc de montrer que si $\chi \in \overline{\widetilde{\mathcal{B}_{F}}}$, alors $\overline{\widetilde{\mathcal{B}_{F}}}=L^{2}(0,1)$.

Supposons $\chi \in \overline{\widetilde{\mathcal{B}_{F}}}$. L'ensemble des fonctions étagées est dense dans $L^{2}(0,1)$, il suffit donc de montrer que toute fonction étagée est limite d'une suite de $\widetilde{\mathcal{B}_{F}}$ dans $L^{2}(0,1)$. La fonction $D_{\alpha}(\chi)$ est colinéaire à la fonction indicatrice de l'intervalle ] $0, \alpha]$ donc toute fonction étagée sur ]0,1] est, presque partout une combinaison linéaire des $\left.\left.D_{\alpha}(\chi), \alpha \in\right] 0,1\right]$. Il suffit donc de montrer que pour tout $\left.\left.\alpha \in\right] 0,1\right]$, $D_{\alpha}(\chi) \in \overline{\widetilde{\mathcal{B}_{F}}}$, ce que nous savons car $\widetilde{\widetilde{\mathcal{B}}_{F}}$ est stable par l'action de $\left.\left.\left\{D_{\alpha}, \alpha \in\right] 0,1\right]\right\}$.

On peut étendre le théorème $\llbracket$ à l'espace $\mathcal{B}_{F}$. L'idée de la démonstration de ce second théorème a été fournie par J.-F. Burnol, que je remercie.

Théorème II. Soit $F$ une fonction vérifiant les hypothèses 11 telle que $\Psi_{F}^{(1)} \in$ $L^{2}(0,+\infty)$. Alors les assertions suivantes sont équivalentes :

(1) La fonction $F$ ne s'annule pas dans le demi-plan $\Re s>1 / 2$;

(2) le sous-espace $\mathcal{B}_{F}$ est dense dans $L^{2}(0,1)$;

(3) la fonction indicatrice $\chi$ de l'intervalle ]0,1] appartient à l'adhérence de $\mathcal{B}_{F}$ dans $L^{2}(0,1)$. 
Démonstration. Au vu du théorème [ il nous suffit de montrer que si $F$ admet un zéro de partie réelle supérieure à $1 / 2$, alors $\chi$ n'appartient pas à l'adhérence de $\mathcal{B}_{F}$. Soit $\rho$ un zéro de $F$ tel que $\Re \rho>1 / 2$.

Soit $f(t)=\sum_{k=1}^{n} c_{k} \Psi_{F}^{(1)}\left(\frac{t}{\alpha_{k}}\right) \in \mathcal{B}_{F}$. On a pour $1 / 2<\Re s<1, \mathcal{M} f(s)=$ $-\frac{F(s)}{s} g(s)$, où $g(s)=\sum_{k=1}^{n} c_{k} \alpha_{k}^{s}$.

D'autre part, pour $\Re s>0, \mathcal{M} \chi(s)=\frac{1}{s}$.

Nous allons montrer que l'on ne peut pas approcher $\frac{1}{s}$ par des fonctions de la forme $\frac{F(s)}{s} g(s)$ avec $g(s)=\sum_{k=1}^{n} c_{k} \alpha_{k}^{s}, 0<\alpha_{k} \leq 1, c_{k} \in \mathbb{C}$.

Posons $u_{\rho}(s)=\frac{1}{1-\bar{\rho}-s}\left(\frac{s}{s-1}\right)^{m_{F}}$.

La fonction $u_{\rho}$ appartient à l'espace $L^{2}(0,+\infty)$ car pour $s=\frac{1}{2}+i t$,

$$
\left|u_{\rho}(s)\right|=\frac{1}{\sqrt{\left(\frac{1}{2}-\Re \rho\right)^{2}+(\Im \rho-t)^{2}}} .
$$

Calculons le produit hilbertien de $\frac{F g}{s}$ par $u_{\rho}$ :

$$
\left\langle\frac{F g}{s}, u_{\rho}\right\rangle=\int_{\frac{1}{2}-i \infty}^{\frac{1}{2}+i \infty} \frac{F(s) g(s)}{s}\left(\frac{s-1}{s}\right)^{m_{F}} \frac{1}{s-\rho} d s .
$$

Or la fonction $\frac{F(s) g(s)}{s}\left(\frac{s-1}{s}\right)^{m_{F}}$ appartient à l'espace $L^{2}(1 / 2+i \mathbb{R})$ donc on a :

$$
\left\langle\frac{F g}{s}, u_{\rho}\right\rangle=2 i \pi \frac{F(\rho) g(\rho)}{\rho}\left(\frac{\rho-1}{\rho}\right)^{m_{F}}=0
$$

D'autre part,

$$
\left\langle\frac{1}{s}, u_{\rho}\right\rangle=\int_{\frac{1}{2}-i \infty}^{\frac{1}{2}+i \infty} \frac{1}{s}\left(\frac{s-1}{s}\right)^{m_{F}} \frac{1}{s-\rho} d s=2 i \pi\left(\frac{\rho-1}{\rho}\right)^{m_{F}} \frac{1}{\rho} \neq 0,
$$

et donc $\frac{1}{s}$ n'est pas dans l'adhérence du sous-espace engendré par les fonction $\frac{F(s) g(s)}{s}$.

On en conclut, en utilisant le théorème de Paley-Wiener que la fonction $\chi$ n'appartient pas à l'adhérence de $\mathcal{B}_{F}$.

Remarque 4. La démonstration du critère de Beurling-Nyman pour le cas $p=$ 2 repose sur des arguments hilbertiens et sur la transposition d'un problème de localisation de zéros à un problème de sous-espaces invariants dans un espace de Hardy. Pour la fonction $\zeta$ de Riemann, Báez-Duarte [BD] affine ce critère. Sa démonstration ne repose pas sur le résultat de Beurling et Lax utilisé ici mais, au contraire, sur des arguments classique de la théorie analytique des nombres. Nous pouvons généraliser ce résulat à la classe de Selberg. Les détails seront explicités dans un travail ultérieur.

3.4. Un critère pour que $\Psi_{F}^{(1)}$ soit de carré intégrable. Le théorème que nous avons obtenu nécessite une hypothèse forte, qui est l'appartenance de la fonction $\Psi_{F}^{(1)}$ à l'espace $L^{2}(0,+\infty)$. Nous allons préciser ce que cette condition signifie et donner un critère pour qu'elle soit vérifiée. Nous donnerons dans le cas de la classe de Selberg une version "lissée" de notre théorème afin de supprimer cette condition.

Nous utiliserons le théorème de Hardy, Littlewood et Ingham suivant pour démontrer notre critère. C'est le théorème 7 de l'article [HIP]. 
Lemme 1 (Théorème de Hardy, Littlewood et Ingham). Soient $D=\{\alpha<\Re z<\beta\}$ une bande verticale et $f$ une fonction continue sur $\bar{D}$, analytique dans $D$ et telle qu'il existe un réel $k$ vérifiant $f(z)=O\left(e^{e^{k|\Im z|}}\right)$ pour tout $z \in D$.

Supposons que pour $x=\alpha$ et $x=\beta$, l'intégrale $J(x)=\int_{-\infty}^{+\infty}|f(x+i y)|^{2} d y$ converge. Alors:

(1) $J(x)$ converge pour $\alpha<x<\beta$;

(2) $J(x) \leq \max (J(\alpha), J(\beta))$;

(3) $\log J(x)$ est convexe.

Proposition 3.3. Si F est une fonction vérifiant les hypothèses 1, alors :

$$
\Psi_{F}^{(1)} \in L^{2}(0,+\infty) \Leftrightarrow \frac{F\left(\frac{1}{2}+i \tau\right)}{\frac{1}{2}+i \tau} \in L^{2}(\mathbb{R}) .
$$

Démonstration. Remarquons tout d'abord qu'en utilisant (3.2) et la définition de $\Psi_{F}$, on a

$$
\Psi_{F}^{(1)} \in L^{2}(0,+\infty) \Leftrightarrow \int_{0}^{1}\left|\Psi_{F}^{(1)}(x)\right|^{2} d x<+\infty .
$$

Notons $\alpha$ l'abscisse de convergence absolue de l'intégrale $\int_{1}^{+\infty} \Psi_{F}(t) t^{-s-1} d t$; Pour tout $\varepsilon>0, \Psi_{F}(x) \ll x^{1+\varepsilon}$ et donc $\alpha \leq 1$.

Pour $\Re s>\alpha$, posons

$$
G(s)=\int_{0}^{1} \Psi_{F}^{(1)}(x) x^{s-1} d x=\int_{1}^{+\infty} \Psi_{F}(t) t^{-s-1} d t .
$$

D'après (3.4), pour $\Re s>1$, on a

$$
-\frac{F(s)}{s}+A(s)=G(s)=\int_{0}^{1} \Psi_{F}^{(1)}(u) u^{s-1} d u .
$$

Cette dernière fonction est la transformée de Mellin de $\Psi_{F}^{(1)} \chi$ et est donc holomorphe dans son demi-plan de convergence absolue $\{\Re s>\alpha\}$. D'autre part, la fonction $-\frac{F(s)}{s}+A(s)$ est holomorphe dans le demi-plan $\{\Re s \geq 1 / 2\}, G$ se prolonge donc holomorphiquement à $\{\Re s \geq 1 / 2\}$. Par unicité du prolongement analytique, l'égalité (3.7) est valable dans le demi-plan $\{\Re s>\max (\alpha, 1 / 2)\}$.

- Si $\Psi_{F}^{(1)} \in L^{2}(0,+\infty)$, alors $\Psi_{F}^{(1)} \chi \in L^{2}(0,1)$ et d'après le théorème de PaleyWiener, $G \in H^{2}$.

La limite non-tangentielle $G^{*}$ de $G$, définie sur $\mathbb{R}$ par

$$
G^{*}(y)=\lim _{x \rightarrow \frac{1}{2}} G(x+i y),
$$

appartient donc à $L^{2}(\mathbb{R})$.

Comme $G$ est holomorphe dans $\{\Re s \geq 1 / 2\}$, on a:

$$
G^{*}(y)=G\left(\frac{1}{2}+i y\right) \in L^{2}(\mathbb{R})
$$

et donc

$$
\frac{F\left(\frac{1}{2}+i y\right)}{\frac{1}{2}+i y} \in L^{2}(\mathbb{R})
$$


- Réciproquement, si $\frac{F\left(\frac{1}{2}+i y\right)}{\frac{1}{2}+i y} \in L^{2}(\mathbb{R})$, alors $G\left(\frac{1}{2}+i y\right) \in L^{2}(\mathbb{R})$.

Nous allons appliquer le lemme 1 à $G$.

La fonction $G$ est analytique dans $\{\Re s \geq 1 / 2\}$ d'ordre fini. D'autre part les fonctions $\tau \mapsto G\left(\frac{1}{2}+i \tau\right)$ et $\tau \mapsto G(2+i \tau)$ sont des fonctions de $L^{2}(\mathbb{R})$ car la fonction $F(2+i \tau)$ est bornée.

Ainsi, d'après le lemme 1, pour tout

$$
x \in] \frac{1}{2}, 2[,
$$

l'intégrale $\int_{-\infty}^{+\infty}|G(x+i \tau)|^{2} d \tau$ converge et

$$
\int_{-\infty}^{+\infty}|G(x+i \tau)|^{2} d \tau \leq \max \left(\int_{-\infty}^{+\infty}\left|G\left(\frac{1}{2}+i \tau\right)\right|^{2} d \tau, \int_{-\infty}^{+\infty}|G(2+i \tau)|^{2} d \tau\right) .
$$

Comme de plus l'intégrale $\int_{-\infty}^{+\infty}|G(x+i \tau)|^{2} d \tau$ converge uniformément par rapport à $x \geq \frac{3}{2}$, on a bien $G \in H^{2}$.

Comme $H^{2}=\mathcal{M}\left(L^{2}(0,1)\right)$, il existe $\phi \in L^{2}(0,1)$ telle que $G=\mathcal{M} \phi$. On a alors pour $\Re s>1, G(s)=\mathcal{M} \phi(s)=\mathcal{M}\left(\Psi_{F}^{(1)} \chi\right)(s)$. Par injectivité de la transformation de Mellin, on a donc $\Psi_{F}^{(1)} \chi=\phi$ donc $\Psi_{F}^{(1)} \chi \in L^{2}(0,1)$, donc $\Psi_{F}^{(1)} \in L^{2}(0,+\infty)$.

\section{Lien aVec L'hypothèse de Riemann GÉNÉRALisée}

Nous allons maintenant énoncer le théorème de Beurling-Nyman pour une fonction de la classe de Selberg.

4.1. La classe de Selberg. La classe de Selberg a été introduite en 1989 dans Sel]. Pour un survol des résultats connus sur cette classe de fonction, on pourra consulter [KP0. Conjecturalement, cette classe est l'ensemble des fonctions $L$ de formes automorphes. On sait inconditionnellement qu'elle contient des fonctions jouant un rôle essentiel en théorie des nombres telles que la fonction $\zeta$ de Riemann, les fonctions $L$ de Dirichlet associées à un caractère primitif, les fonctions $\zeta$ de Dedekind associées à un corps de nombres, les fonctions $L$ d'Artin abéliennes,....

Définition 4.1. On dira qu'une fonction $F$ appartient à la classe de Selberg $S$ si $F$ vérifie les conditions suivantes :

(1) Pour $\Re s>1, F(s)=\sum_{n=1}^{\infty} \frac{a(n)}{n^{s}}$ est une série de Dirichlet absolument convergente;

(2) il existe un entier naturel $m$ tel que $(s-1)^{m} F(s)$ soit une fonction entière d'ordre fini;

(3) la fonction $F$ satisfait une équation fonctionnelle de la forme :

$$
\Phi(s)=\omega \overline{\Phi(1-\bar{s})} \text { où } \Phi(s)=\alpha Q^{s} \gamma(s) F(s),
$$

avec $\gamma(s)=\prod_{j=1}^{r} \Gamma\left(\lambda_{j} s+\mu_{j}\right), \lambda_{j}>0, \Re \mu_{j} \geq 0, Q>0$ et $|\alpha|=1 ;$

(4) pour tout $\varepsilon>0, a(n)=O\left(n^{\varepsilon}\right)$;

(5) pour $\Re s$ assez grand,

$$
\log F(s)=\sum_{n=1}^{+\infty} b_{n} n^{-s}
$$


où $b_{n}=0$ si $n$ n'est pas une puissance d'un nombre premier et $b_{n}=O\left(n^{\theta}\right)$ pour un $\theta<1 / 2$.

On notera $d=2 \sum_{j=1}^{r} \lambda_{j}$, que l'on appellera degré de $F$.

On distinguera parmi les zéros de $F$ les zéros de la forme $-\frac{n+\mu_{j}}{\lambda_{j}}, n \in \mathbb{N}, j \in$ [1, $r]$, que l'on appelera zéros triviaux de $F$. Remarquons que les assertions (1) et (4) montrent que les fonctions de la classe de Selberg ne s'annulent pas dans le demi-plan $\Re s>1$ et grâce à la symétrie induite par l'équation fonctionnelle, les zéros d'une fonction $F$ de la classe de Selberg non triviaux, sont de parties réelles comprises entre 0 et 1 . Ils sont de plus répartis symétriquement par rapport à la droite $\Re s=1 / 2$ que l'on appelera droite critique.

Conjecture 1. On conjecture que les zéros non triviaux de F sont de partie réelle égale à $1 / 2$, c'est ce que l'on appelle hypothèse de Riemann généralisée pour la fonction $F$ et nous la noterons désormais ( $H R G)$.

Notons qu'en utilisant la symétrie induite par l'équation fonctionnelle, cette conjecture est équivalente à la non-annulation de la fonction $F$ dans le demi-plan $\Re s>1 / 2$.

4.2. Majoration d'une fonction de la classe de Selberg; hypothèse de Lindelöf généralisée. Nous rappelons ici des majorations classiques des fonctions de la classe de Selberg. La démonstration utilise la formule de Stirling complexe que l'on applique au produit de fonctions $\Gamma$ qui apparait dans l'équation fonctionnelle et le principe de Phragmèn-Lindelöf ( $\$ 5.65$ de [T1]).

Proposition 4.1. - Soit $\delta>0$. Alors on a uniformément pour $\Re s \geq 1+\delta$

$$
|F(s)|=O_{\delta}(1) .
$$

- Pour $\sigma<0$, on a

$$
F(\sigma+i \tau)=O_{\sigma}\left((1+|\tau|)^{d(1 / 2-\sigma)}\right) .
$$

- Pour $0 \leq \sigma \leq 1$ et pour tout $\varepsilon>0$, on a

$$
F(s)=O_{\sigma, \varepsilon}\left((1+|\tau|)^{\frac{d}{2}(1-\sigma)+\varepsilon}\right) .
$$

Pour tout $\varepsilon>0$, pour tout $0<\sigma_{1}<\sigma_{2}<1$, il existe $c=c\left(\varepsilon, \sigma_{1}, \sigma_{2}\right)$ tel que pour tout $\sigma_{1} \leq \sigma \leq \sigma_{2}$

$$
F(\sigma+i \tau) \leq c(1+|\tau|)^{\frac{d}{2}\left(1-\sigma_{1}\right)+\varepsilon} .
$$

Conjecture 2. On conjecture que pour tout $\varepsilon>0$,

$$
F(1 / 2+i \tau)=O_{\varepsilon}\left((1+|\tau|)^{\varepsilon}\right) .
$$

C'est ce que l'on appelle hypothèse de Lindelöf généralisée pour la fonction $F$ et nous la noterons désormais $(H L G)$.

Nous allons à présent donner quelques estimations de $F(s)$ dans le cas où la fonction $F$ vérifie l'hypothèse de Riemann généralisée. Pour établir ces estimations, nous avons suivi la méthode que Titchmarsch utilisait dans [T2] pour l'étude de la fonction $\zeta$ de Riemann. 
Proposition 4.2. Soit $F$ une fonction de $S$ vérifiant (HRG).

Soit $\delta$ vérifiant $0<\delta<\frac{1}{2}$. On a uniformément par rapport à $\sigma \geq \frac{1}{2}+\delta$

$$
|\log F(\sigma+i \tau)|=O\left(\delta^{-1} \log (1+|\tau|)\right) .
$$

Démonstration. Soit $\tau \in \mathbb{R}$ tel que $|\tau| \geq 2$. On pose $z_{0}=2+i \tau, R=\frac{3}{2}-\frac{\delta}{2}$ et $r=\frac{3}{2}-\delta$. F vérifie (HRG) donc pour $z$ tel que $\Re\left(z+z_{0}\right)>\frac{1}{2}$ et $z+z_{0} \neq 1$, la fonction $H$ définie par $H(z)=\log \frac{F\left(z+z_{0}\right)}{F\left(z_{0}\right)}$ est holomorphe dans le disque $(|z| \leq R)$. Si $|z|=R$, alors $\Re\left(z+z_{0}\right) \geq \frac{1}{2}+\frac{\delta}{2}$, et donc d'après (4.5)

$$
\Re \log F\left(z+z_{0}\right) \leq\left(\frac{d}{4}+1\right) \log (1+\tau),
$$

d'autre part

$$
\Re \log F\left(z_{0}\right)=\log |F(2+i \tau)| \gg 2^{-1 / 2} .
$$

En effet, si $\theta$ et $b_{n}$ sont les données définie en (4.1), on a

$$
|\log F(2+i \tau)| \leq \sum_{n=2}^{+\infty} \frac{|b(n)|}{n^{2}} \leq \sum_{n=2}^{+\infty} n^{\theta-2}=O\left(2^{\theta-1}\right)=O\left(2^{-1 / 2}\right)
$$

On peut donc appliquer un corollaire du lemme de la partie réelle (cf. p.153 de [Te]) à la fonction $H$ sur les cercles de centre $z_{0}$ et de rayons $\frac{3}{2}-\frac{\delta}{2}$ et $\frac{3}{2}-\delta$. Sur le grand cercle,

$$
\Re H(z) \leq c\left(\log (1+|\tau|)+2^{-1 / 2}\right)
$$

où $c$ est une constante ne dépendant que de $F$. Donc sur le petit cercle

$$
\left|\log F\left(z+z_{0}\right)\right| \leq \frac{3-2 \delta}{\frac{1}{2} \delta} c\left(\log (1+|\tau|)+2^{-1 / 2}\right)
$$

et donc $\left|\log F\left(z+z_{0}\right)\right| \ll \delta^{-1} \log (1+|\tau|)$.

Proposition 4.3. Pour tout $0<\varepsilon<1 / 2$,

$$
\log F(\sigma+i \tau)=O_{\varepsilon}\left((\log (1+|\tau|))^{2-2 \sigma+\varepsilon}\right)
$$

uniformément pour $\frac{1}{2}+\varepsilon \leq \Re s \leq 1$ lorsque $|\tau|$ tend vers l'infini.

Démonstration. Soit $s=\sigma+i \tau, \frac{1}{2}<\sigma \leq 1,|\tau| \geq 2$. Soit $\sigma_{1}>1$ et $0<\varepsilon<$ $\min \left(\sigma_{1}-1, \sigma-\frac{1}{2}\right)$. Posons $R_{1}=\sigma_{1}-1-\varepsilon, r=\sigma_{1}-\sigma, R_{2}=\sigma_{1}-\frac{1}{2}-\varepsilon$. On a donc $R_{1}<r<R_{2}$ et $\left|s-\left(\sigma_{1}+i \tau\right)\right|=r$. Posons $H(w)=\log \left(F\left(s_{0}+w\right)\right)$, où $s_{0}:=\sigma_{1}+i \tau$. La fonction $H$ est holomorphe dans $R_{1} \leq|w| \leq R_{2}$. $H$ vérifie les hypothèses du lemme des trois cercles de Hadamard (cf. p.173 de [Te]). En notant $M(R)=\max _{|w|=R}|H(w)|$, on a donc:

$$
\log M(r) \leq \frac{\log \frac{R_{2}}{r}}{\log \frac{R_{2}}{R_{1}}} \log M\left(R_{1}\right)+\frac{\log \frac{r}{R_{1}}}{\log \frac{R_{2}}{R_{1}}} \log M\left(R_{2}\right) .
$$


Autrement dit, en posant $\alpha=\frac{\log \frac{r}{R_{1}}}{\log \frac{R_{2}}{R_{1}}}$ :

$$
\begin{gathered}
\log |F(s)| \leq M\left(R_{1}\right)^{1-\alpha} M\left(R_{2}\right)^{\alpha}, \\
\alpha=\frac{\log \frac{\sigma_{1}-\sigma}{\sigma_{1}-1-\varepsilon}}{\log \frac{\sigma_{1}-\frac{1}{2}-\varepsilon}{\sigma_{1}-1-\varepsilon}}=\frac{\frac{-\sigma+1+\varepsilon}{\sigma_{1}}+o\left(\frac{1}{\sigma_{1}}\right)}{-\frac{1}{2 \sigma_{1}}-\frac{\varepsilon}{\sigma_{1}}+\frac{1}{\sigma_{1}}+\frac{\varepsilon}{\sigma_{1}}+o\left(\frac{1}{\sigma_{1}}\right)}=\frac{-\sigma+1+\varepsilon+o(1)}{\frac{1}{2}+o(1)}
\end{gathered}
$$

qui tend vers $2(1+\varepsilon-\sigma)$ quand $\sigma_{1}$ tend vers $+\infty$.

$$
M\left(R_{1}\right)=\max _{t \in \mathbb{R}}\left|\log F\left(\sigma_{1}+i \tau+\left(\sigma_{1}-1-\varepsilon\right) e^{i t}\right)\right| \leq \sup _{\substack{\sigma \geq 1+\varepsilon \\ t \in \mathbb{R}}}|\log F(\sigma+i t)| \ll_{\varepsilon} 1,
$$

d'après (4.2) et

$$
M\left(R_{2}\right)=\max _{t \in \mathbb{R}}\left|\log F\left(\sigma_{1}+i \tau+\left(\sigma_{1}-\frac{1}{2}-\varepsilon\right) e^{i t}\right)\right| \ll_{\varepsilon} \log (1+|\tau|),
$$

d'après la proposition 4.2 .

Ainsi $\log |F(s)| \ll_{\varepsilon}(\log (1+|\tau|))^{2-2 \sigma+2 \varepsilon} \quad\left(\frac{1}{2}+\varepsilon \leq \sigma \leq 1,|\tau| \geq 2\right)$.

Proposition 4.4. Soit F une fonction de S. Alors l'hypothèse de Riemann pour $F$ entraîne l'hypothèse de Lindelöf pour $F$.

C'est une conséquence immédiate de la proposition 4.3 .

4.3. Le théorème de Beurling-Nyman pour une fonction de la classe de Selberg. Remarquons que si $F$ est une fonction de $S$ vérifiant l'hypothèse de Lindelöf généralisée, alors la fonction $\frac{F(s)}{s}$ est de carré intégrable sur la droite $\Re s=\frac{1}{2}$ et donc d'après la proposition 3.3 la fonction $\Psi_{F}^{(1)}$ appartient à l'espace $L^{2}(0,+\infty)$. En utilisant le théorème \ et la proposition 4.4, nous sommes donc en mesure de conclure :

Théorème III. Soit F une fonction de la classe de Selberg S. Alors les assertions suivantes sont équivalentes:

(1) La fonction $F$ vérifie l'hypothèse de Riemann généralisée;

(2) $\Psi_{F}^{(1)} \in L^{2}(0,+\infty)$ et le sous-espace $\mathcal{B}_{F}$ est dense dans $L^{2}(0,1)$;

(3) $\Psi_{F}^{(1)} \in L^{2}(0,+\infty)$ et la fonction indicatrice $\chi$ de l'intervalle $\left.] 0,1\right]$ appartient à l'adhérence de $\mathcal{B}_{F}$ dans $L^{2}(0,1)$.

\section{Suppression de la condition D'appartenance À $L^{2}(0,+\infty)$}

Nous avons démontré dans $[\mathrm{dR}$ que pour une fonction $F$ de la classe de Selberg de degré inférieur à 4 , la fonction $\Psi_{F}^{(1)}$ est de carré intégrable.

Cette hypothèse est cependant une hypothèse forte puisque l'on peut montrer que l'hypothèse de Lindelöf généralisée pour la fonction $F$ est équivalente à la condition suivante :

$$
\forall k \in \mathbb{N}, \Psi_{F^{k}}^{(1)} \in L^{2}(0,+\infty) .
$$

Afin de supprimer cette condition, nous allons introduire une fonction complémentaire lissée. Cette idée nous a été suggérée par E. Kowalski que nous tenons à remercier ici. 
Définition 5.1. Soit $F$ une fonction de la classe de Selberg. Pour tout $k \in \mathbb{N}^{*}$, on définit sur $\mathbb{R}^{+*}$ la fonction $\Psi_{k, F}$, fonction complémentaire d'ordre $k$ associée à $F$ par :

$$
\Psi_{k, F}(x)=\operatorname{Res}\left(\frac{F(s) x^{s}}{s(s+1) \cdots(s+k)}, 1\right)-\frac{1}{k !} \sum_{n \leq x} a_{n}\left(1-\frac{n}{x}\right)^{k} .
$$

On définit également la fonction $\Psi_{k, F}^{(1)} \operatorname{par} \Psi_{k, F}^{(1)}(x)=\Psi_{k, F}(1 / x)$.

Remarque 5. Pour $k=0$, on retrouve bien les fonctions $\Psi_{F}$ et $\Psi_{F}^{(1)}$ définies précédemment.

Proposition 5.1. Soit $F \in S$. Si $k>\frac{d}{4}$, alors la fonction $\Psi_{k, F}^{(1)}$ appartient $\grave{a}$ l'espace $L^{2}(0,+\infty)$ et pour $\max \left(0,1-\frac{2 k}{d}\right)<\Re s<1$, on a :

$$
\mathcal{M} \Psi_{k, F}^{(1)}(s)=\frac{F(s)}{s(s+1) \cdots(s+k)} .
$$

Démonstration. En posant $A^{k}(x)=\frac{1}{k !} \sum_{n \leq x} a_{n}(x-n)^{k}$, on a :

$$
\Psi_{k, F}(x)=\operatorname{Res}\left(\frac{F(s) x^{s}}{s(s+1) \cdots(s+k)}, 1\right)-x^{-k} A^{k}(x) .
$$

D'après un calcul classique de transformée de Mellin inverse, on a pour tout $c>1$ :

$$
A^{k}(x)=\frac{1}{2 i \pi} \int_{\Re s=c} \frac{F(s) x^{s+k}}{s(s+1) \cdots(s+k)} d s .
$$

D'après (4.4), pour tout $\varepsilon>0$, on a $|F(s)| \ll(1+|\tau|)^{\frac{d}{2}(1-\Re s)+\varepsilon}$, donc pour $\max \left(0,1-\frac{2 k}{d}\right)<c^{\prime}<1$, l'intégrale $\int_{\Re s=c^{\prime}} \frac{F(s) x^{s}}{s(s+1) \cdots(s+k)} d s$ converge et on a

$$
\Psi_{k, F}(x)=\frac{1}{2 i \pi} \int_{\Re s=c^{\prime}} \frac{F(s) x^{s}}{s(s+1) \cdots(s+k)} d s .
$$

On a donc pour tout $\varepsilon>0$, lorsque $x$ tend vers l'infini

$$
\Psi_{k, F}(x)=O\left(x^{\max \left(0,1-\frac{2 k}{d}\right)+\varepsilon}\right) .
$$

D'autre part on peut montrer que pour $x<1$,

$$
\Psi_{k, F}(x)=\operatorname{Res}\left(\frac{F(s) x^{s}}{s(s+1) \cdots(s+k)}, 1\right)=x Q_{F}(\ln x),
$$

où $Q_{F}$ est un polynôme de degré $m_{F}-1$.

On a donc pour tout $\varepsilon>0$, lorsque $x$ tend vers 0 ,

$$
\Psi_{k, F}(x)=O\left(x^{1-\varepsilon}\right) .
$$

D'après (5.2) et (5.3), en supposant $k>d / 4$, la fonction $\Psi_{k, F}^{(1)}$ appartient à $L^{2}(0,+\infty)$ et pour $\max \left(0,1-\frac{2 k}{d}\right)<\Re s<1$, on a :

$$
\mathcal{M} \Psi_{k, F}^{(1)}(s)=\frac{F(s)}{s(s+1) \cdots(s+k)} .
$$


Définissons à présent le sous-espace $\widetilde{\mathcal{B}_{k, F}}$.

Définition 5.2. Soit $F$ une fonction de la classe de Selberg. On définit les espaces suivants :

$$
\mathcal{B}_{k, F}=\left\{f: t \mapsto \sum_{k=1}^{n} c_{k} \Psi_{k, F}\left(\frac{\alpha_{k}}{t}\right), n \in \mathbb{N}, \forall k \in[1, n], c_{k} \in \mathbb{C}, 0<\alpha_{k} \leq 1\right\}
$$

et

$$
\widetilde{\mathcal{B}_{k, F}}=\left\{f: t \mapsto \sum_{k=1}^{n} c_{k} \Psi_{k, F}\left(\frac{\alpha_{k}}{t}\right) \in \mathcal{B}_{k, F}, c_{k} \text { et } \alpha_{k} \text { vérifiant (A) }\right\}
$$

où

$$
\forall l \in\left[\left[0, m_{F}-1\right]\right], \sum_{k=1}^{n} c_{k} \alpha_{k}\left(\ln \alpha_{k}\right)^{l}=0 .
$$

Si $k>\frac{d}{4}$, alors $\Psi_{k, F}^{(1)} \in L^{2}(0,+\infty)$ et si $f$ est une fonction de $\mathcal{B}_{k, F}$ de la forme

$$
f(t)=\sum_{k=1}^{n} c_{k} \Psi_{k, F}\left(\frac{\alpha_{k}}{t}\right)
$$

alors pour $\max \left(0,1-\frac{2 k}{d}\right)<\Re s<1$, on a :

$$
\mathcal{M} f(s)=g(s) \frac{F(s)}{s(s+1) \cdots(s+k)},
$$

avec $g(s)=\sum_{k=1}^{n} c_{k} \alpha_{k}^{s}$. De plus, les espaces $\mathcal{B}_{k, F}$ et $\widetilde{\mathcal{B}_{k, F}}$ sont des sous-espaces de $L^{2}(0,+\infty)$ et $L^{2}(0,1)$ respectivement.

En suivant exactement la démonstration du théorème【, on montre donc que $\widetilde{\mathcal{B}_{k, F}}$ est un sous-espace de $L^{2}(0,1)$ invariant par l'action du semi-groupe des contractions $\left\{D_{\theta}, 0<\theta \leq 1\right\}$, contenant la fonction $\Psi_{k, F}^{(1)}$. Son image par la transformation de Mellin est donc un sous-espace invariant de $H^{2}$. On peut donc appliquer le théorème de Beurling-Lax à son adhérence et on obtient comme précédemment $\overline{\mathcal{M}} \overline{\mathcal{B}_{k, F}}=B_{Z_{F}} H^{2}$. Finalement, on obtient le théorème suivant.

Théorème IV. Soit $F$ une fonction de la classe de Selberg. Soit $k$ un entier vérifiant $k>\frac{d}{4}$. Alors les assertions suivantes sont équivalentes :

(1) La fonction $F$ vérifie l'hypothèse de Riemann généralisée;

(2) le sous-espace $\widetilde{\mathcal{B}_{k, F}}$ est dense dans $L^{2}(0,1)$;

(3) la fonction indicatrice $\chi$ de l'intervalle ]0,1] appartient à l'adhérence de $\widetilde{\mathcal{B}_{k, F}}$ dans $L^{2}(0,1)$.

Ce théorème reste valable en remplaçant $\widetilde{\mathcal{B}_{k, F}} \operatorname{par} \mathcal{B}_{k, F}$.

En remplaçant la fonction complémentaire par sa version lissée, on obtient donc un théorème qui ne nécessite plus d'hypothèse puissante.

\section{REFERENCES}

[BD] L. Báez-Duarte, A strengthening of the Nyman-Beurling criterion for the Riemann Hypothesis, Rendiconti Accad. Lincei 23 (2003), 5-11. MR2057270 (2005b:11135)

[BS] M. Balazard, E. Saias, The Nyman-Beurling equivalent form for the Riemann hypothesis, Expo. Math. 18 (2000), 131-138. MR1759315 (2001i:11109) 
[BF] H. Bercovici, C. Foias, A real variable restatement of Riemann's hypothesis, Israel J. of Maths. 48 (1984), 57-68. MR768266 (86d:11065)

[Be] A. Beurling, A closure problem related to the Riemann Zeta-function, Proc. Nat. Acad. Sci. 41 (1955), 312-314. MR0070655 (17:15a)

[CG1] J. B. Conrey, A. Ghosh, Remarks on the generalized Lindelöf Hypothesis, Preprint.

[HIP] G. H. Hardy, A. E. Ingham, G. Pólya, Theorems concerning mean values of analytic functions, Proc. of the Royal Soc. 113 (1927), 542-569.

[KP0] J. Kaczorowski, A. Perelli, The Selberg class: a survey, Acta Math. 192 (1999), 953-992. MR:1689554 (2001g:11141)

[Lev] N. Levinson, Gap and Density Theorems, A.M.S. Coll. Publ. XXVI, 1940. MR0003208 $(2: 180 d)$

[PW] R. E. A. C. Paley, N. Wiener, The Fourier Transform in the Complex Domain, A.M.S. Coll. Publ. XIX, 1934.

[dR] A. de Roton, On the mean square of an error term for an extended Selberg's class, Acta Arith. 126 (2007), no. 1, 27-55. MR2284311

[Sch] L. Schwartz, Étude des sommes d'exponentielles, Hermann, Paris, 1959. MR0106383 $(21: 5116)$

[Sel] A. Selberg, Old and new conjectures and results about a class of Dirichlet series, Proceedings of the Almafi Conference on Analytic Number Theory, Università di Salerno (1992). MR.1220477(94f:11085)

[Sz] O. Szász, Uber die Approximation stetiger Funktionnen durch lineare Aggregate von Potenzen, Math. Ann. 77 (1916), 482-496. MR.1511875

[Te] G. Tenenbaum, Introduction à la théorie analytique et probabiliste des nombres, Société mathématique de France, 1995. MR.1366197 (97e:11005a)

[T1] E. C. Titchmarsh, The theory of functions, Second edition, Oxford University Press, 1939. MR $882550(88 \mathrm{c}: 11049)$

[T2] E. C. Titchmarsh, The theory of the Riemann Zeta-function, Oxford University Press, 1951. MR0046485 (13:741c)

IECN - Université Henri Poincaré: Nancy 1, BP 239, 54506 Vandoeuvre-lès-Nancy, FRANCE

E-mail address: deroton@iecn.u-nancy.fr 